\title{
Fatigue damage formation and microstructure of ultrafine grained copper under two-step loading
}

\author{
M. Goto ${ }^{1}$, S. Z. $\operatorname{Han}^{2}$, K. $\operatorname{Euh}^{2}$, N. Teshima ${ }^{3}$, T. Yakushiji ${ }^{3}$ \\ \& N. Kawagoishi ${ }^{4}$ \\ ${ }^{1}$ Department of Mechanical Engineering, Oita University, Japan \\ ${ }^{2}$ Korea Institute of Materials Science, Republic of Korea \\ ${ }^{3}$ Oita National College of Technology, Japan \\ ${ }^{4}$ Dai-ichi Institute of Technology, Japan
}

\begin{abstract}
In order to study the effect of stress change on the surface damage formation of ultrafine grained copper, two step fatigue stress tests were conducted. The microstructural evolution and crack growth direction of ultrafine-grained copper fatigued under constant stressing depends on the magnitude of the applied stress amplitude. The growth direction occurred perpendicular to the loading axis at low stresses, and changed to $45^{\circ}$ to the loading axis at high stresses. In the case of high-to-low block stressing, the $45^{\circ}$ inclined growth direction under high stress changed to the perpendicular direction under subsequent low stress. In low-to-high block stressing, although the growth direction perpendicular to the loading axis at low stresses was still retained at subsequent high stress, the degree of zigzag behaviour in the crack growth drastically increased after the stress change. The sizes and topographies of evolved microstructures, which have a great effect on the crack path formation, depended on stress histories (order, cycle number and magnitude of the applied stress). The formation behaviour of the fatigue damage under high- and low-stress amplitudes and the effect of pre-stressing on crack growth direction are discussed from the viewpoints of the microstructural evolution under different stress histories.
\end{abstract}

Keywords: ultrafine grain, copper, fatigue, two-step loading, surface damage. 


\section{Introduction}

Equal channel angular pressing (ECAP) is frequently used to obtain ultrafine grained materials without a decrease in the bulk sizes of material after the severe plastic deformation [1, 2]. For envisaged structural applications of UFG metals, attention has been paid to fatigue performance regarding cyclic properties, S-N characteristics, and formation of shear bands (SBs) [3-8]. To evaluate the fatigue damage, the growth behaviour should be clarified. Fatigue crack propagation in the high-cycle fatigue (HCF) regime has recently attracted great interest. In the HCF regime, the growth behaviour of millimeter-range cracks in UFG metals has been studied using compact tension (CT) [9], and single edge-notched specimens [10]. The crack growth direction of most of those specimens was nearly perpendicular to the loading axis; however, the positional relationship between the specimen faces and ECAP pressing direction was not clearly defined. In strain-controlled low-cycle fatigue (LCF) tests, however, crack growth direction in many UFG metals was oriented at $45^{\circ}$ to the loading axis parallel to the longitudinal axis of the pressed samples [11].

Meanwhile, UFG materials processed by SPD technique have non-equilibrium microstructures [12] with limited thermal and mechanical stability. Such nonequilibrium microstructures can easily change properties under applied cyclic stressing at a certain temperature. Remarkable grain coarsening as a result of dynamic recrystallization has generally occurred in post-fatigued high-purity UFG copper. Since the grain coarsening strongly depends on both the magnitudes and cycle numbers of applied stresses, studies on the microstructural evolution due to various stress histories and its effect on crack growth behaviour are needed for the application of UFG copper to actual machine components used under service loading, because the fatigue crack growth behaviour is dominantly influenced by microstructural inhomogeneities. To date however, such studies are quite few, and certain questions remain unanswered.

To clarify the relationship between fatigue damage and fatigue-induced microstructural evolution, fatigue tests with constant and two-step loading was conducted. The objective of this paper is to discuss the fatigue damage formation and crack growth behaviour, from the viewpoints of the fatigue-induced damage under different stress histories. In addition, the effect of pre-stressing on subsequent growth path formation was investigated.

\section{Experimental procedures}

Pure oxygen-free copper (99.99 wt\% Cu) was used in the experiment. Prior to ECAP processing, the materials were annealed at $500^{\circ} \mathrm{C}$ for $1 \mathrm{hr}$ (average grain size, $100 \mu \mathrm{m})$. Fig. 1(a) shows a schematic of the ECAP die used in this investigation, and the orientation of fatigue specimens relative to the final pressing direction. The die had a $90^{\circ}$ angle between intersecting channels. The angles at the inner and outer corners of the channel intersection were $90^{\circ}$ and $45^{\circ}$, respectively. Repetitive ECAP was accomplished according to the Bc route. Eight extrusion passes resulted in an equivalent shear strain of about 7.8. 

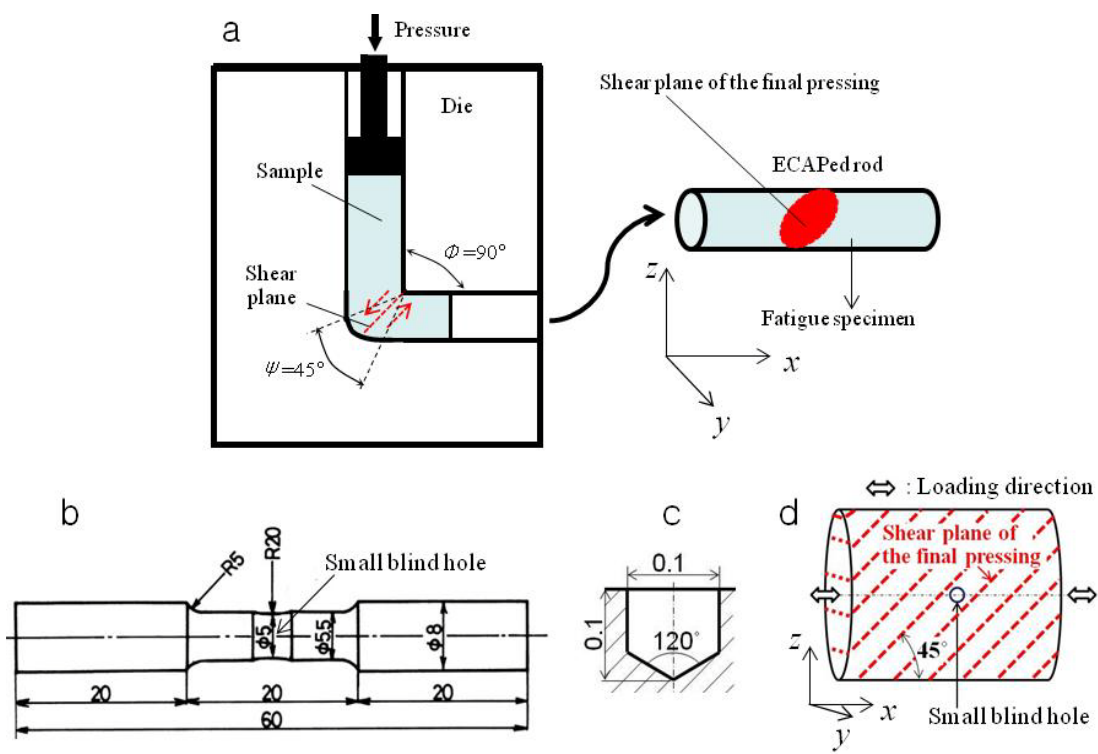

Figure 1: ECAP processing and the fatigue specimen: (a) ECAP process overview and the definition of orientation of specimens relative to the pressing direction; (b) specimen; (c) hole; (d) an illustration showing the location of a hole with respect to the final shear plane.

Fig. 2 shows the inverse polar figure (IPF) map and grain boundary (GB) map of an ECAP rod. The microstructure included fine equiaxed grains with about 300-nm diameter, and large elongated grains. The pre-ECAP mechanical properties were $232 \mathrm{MPa}$ tensile strength, 65\% elongation, and Vickers hardness of 63 (load: $2.9 \mathrm{~N}$ ). After eight ECAP passages, the properties changed to $438 \mathrm{MPa}, 28 \%$, and 141, respectively.

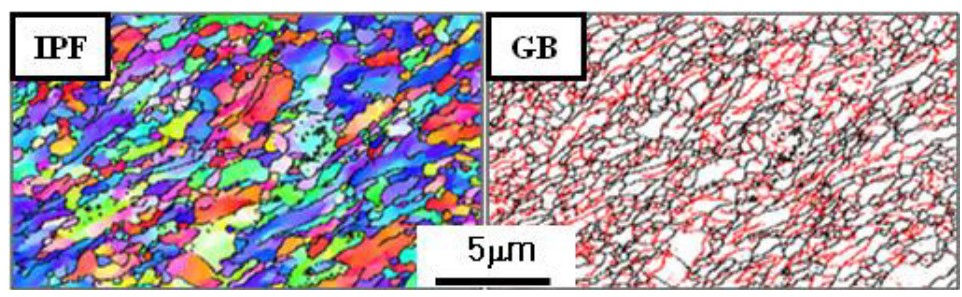

Figure 2: $\quad$ The OIM orientation and GB maps for as-receive UFG copper.

Round bar specimens (Fig. 1(b)) that were $5 \mathrm{~mm}$ in diameter were machined from their respective processed bars. Although the specimens had shallow circumferential notches (20-mm notch radius and $0.25-\mathrm{mm}$ notch depth), the fatigue strength reduction factor for this geometry was close to 1 , meaning that they could be considered plain. The fatigue specimens were electrolytically 
polished ( $\approx 25 \mu \mathrm{m}$ from the surface layer) prior to mechanical testing to remove any preparation-affected surface layer. Prior to testing, a small blind hole (diameter and depth were both $0.1 \mathrm{~mm}$; Fig. 1(c)) was drilled as a crack starter on the middle surfaces of the plain specimens. Fig. 1(d) shows the location of the drilling hole. A hole was drilled on the surface ( $Z X$-plane) where an intersection between the shear plane of the final pressing and the specimen surface makes an angle of $45^{\circ}$ with respect to the loading axis.

All fatigue tests were performed at room temperature using a rotating-bending fatigue machine operating at $50 \mathrm{~Hz}$. The fatigue damage on the specimen surface was observed using optical microscopy (OM) and scanning electron microscopy (SEM). The crack length, $l$, is a length measured along the circumferential direction of the surface. The crack length was measured using a plastic replication technique. The stress value referred to is that of the nominal stress amplitude, $\sigma_{a}$, at the minimum cross-section (5-mm diameter).

\section{Experimental results and discussion}

Fig. 3 shows SEM micrographs of typical surface damage of the specimens fatigued under constant stressing. The surface after the constant stressing of $\sigma_{a}=$ $240 \mathrm{MPa}$ (Fig. 3(a)) consisted of fine line-like protrusions formed at early fatigue stages, and island-like protruded areas formed at later fatigue stages [13]. The line-like protrusions are straight and approximately parallel to the shear plane of the final pressing (as indicated by the arrow). This morphology of protrusions would place them into the persistent slip bands (PSB)-like SBs category [6]. At constant stressing of $\sigma_{a}=90 \mathrm{MPa}$ (Fig. 3(b)), the post-fatigued surface was covered with island-like damaged areas, and the diameter of large areas far exceeded $0.1 \mathrm{~mm}$. The damaged areas contained a high density of fine/shallow protrusions.

To clarify the physical basis of different features of surface damage between the high- and low-stress amplitudes, two-step fatigue stress tests were conducted. After the repetitions of an arbitrary cycle-number at the first stress amplitude $\left(\sigma_{a 1}\right)$, the specimens were fatigued at the second-stress amplitude $\left(\sigma_{a 2}\right)$. Figs. 4(a) and (b) show the surface damage under low-to-high and high-to-low block (L-HB
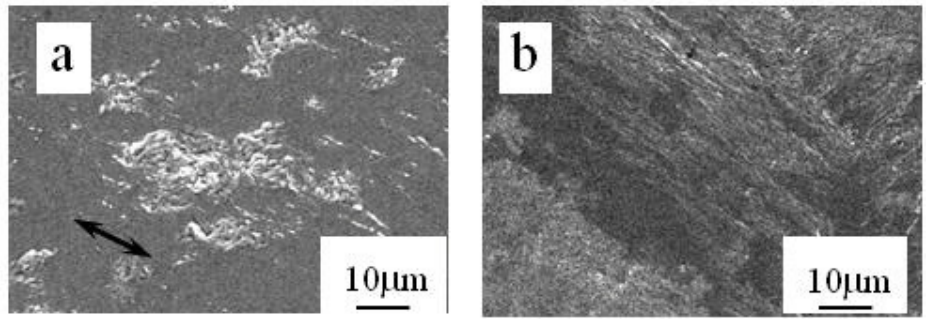

Figure 3: SEM micrographs of surface damage of specimens fatigued under constant stress amplitudes; (a) $\sigma_{a}=240 \mathrm{MPa}$, (b) $\sigma_{a}=90 \mathrm{MPa}$.

Shear direction of the final pressing. 

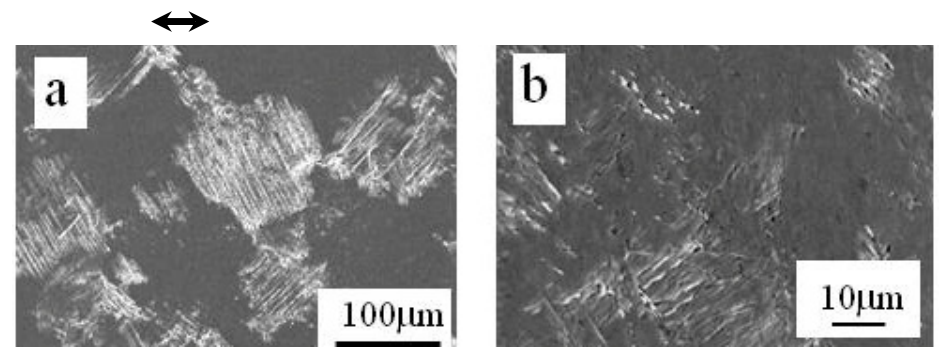

Figure 4: $\quad$ SEM micrographs of surface damage of specimens fatigued under two-step loadings; (a) $\sigma_{a}=90 \rightarrow 240 \mathrm{MPa}$, (b) $\sigma_{a}=240 \rightarrow 90 \mathrm{MPa}$.

and H-LB) stress. In L-HB stressing (Fig. 4(a)), after $2.2 \times 10^{6}$ repetitions of $\sigma_{a 1}=$ $90 \mathrm{MPa}$ (at this stage, a crack from a hole reached $0.59 \mathrm{~mm}$ in length), the specimen was fatigued at $\sigma_{a 2}=240 \mathrm{MPa}$. Fatigued surfaces under L-HB stressing had island-like damaged areas with size ranging from 10 to $100 \mu \mathrm{m}$. A magnified view of the damaged area indicated that slip-bands developed inside the coarsened grain. In H-LB stressing (Fig. 4(b)), after $5.3 \times 10^{4}$ repetitions of $\sigma_{a 1}=$ $240 \mathrm{MPa}$ (at this stage, a crack from a hole reached $0.53 \mathrm{~mm}$ in length), the specimen was fatigued at $\sigma_{a 2}=90 \mathrm{MPa}$. For the H-LB stressing, PSB-like SBs formed under the first high-stress repetitions, and the plate-like protrusions that formed under the subsequent low-stress repetitions were distributed throughout the fatigued surface. The plate-like protrusions exit the matrix at an incline, forming a terraced field of overlapping plate-like protrusions. The sizes of each area covered with plate-like protrusions were roughly less than 10 micrometers.

The EBSD analysis of post-fatigued specimen was conducted to clarify the evolution of microstructure with sub-micron ranges. In order to conduct EBSD analysis of the post-fatigued microstructure just under the specimen surface, $0.15 \mathrm{~mm}$ surface layer of post-fatigued round bar specimens were polished off to make a flat surface for EBSD analysis. The procedure for making the flat surface and the area analyzed by EBSD are illustrated in Fig. 5. Microstructures about $0.7 \mathrm{~mm}$ away from the crack initiation site (at the edge of a small hole) were analyzed.

Fig. 6 shows inverse pole figure (IPF) maps and GB maps of ((a) and (b)) samples fatigued with constant stressing of $\sigma_{a}=240$ and $90 \mathrm{MPa}$, and ((c) and (d)) samples fatigued two-step L-HB and H-LB stressing. The maps for fatigued samples were taken from regions adjacent to crack paths. The GBs in GB maps are denoted either by red lines corresponding to low-angle GBs (LAGBs), where the misorientation, $\theta$, is between $2^{\circ}$ and $15^{\circ}$, or by black lines corresponding to HAGBs with $\theta>15^{\circ}$. The post-fatigue microstructure subjected to the constant stressing experienced grain coarsening, but the coarse grain sizes depended on the magnitude of applied stress amplitudes. At high stress $\left(\sigma_{a}=240 \mathrm{MPa}\right)$, coarse grain evolved, ranging from 1 to 10 micrometers (Fig. 6(a)). The coarse grain sizes are nearly equivalent to the lengths of PSB-like SBs and island-like damaged areas. At low stress amplitude $\left(\sigma_{a}=90 \mathrm{MPa}\right)$, long-term repetitions produced large coarse grains in excess of several tens of micrometers (Fig. 6(b)). 

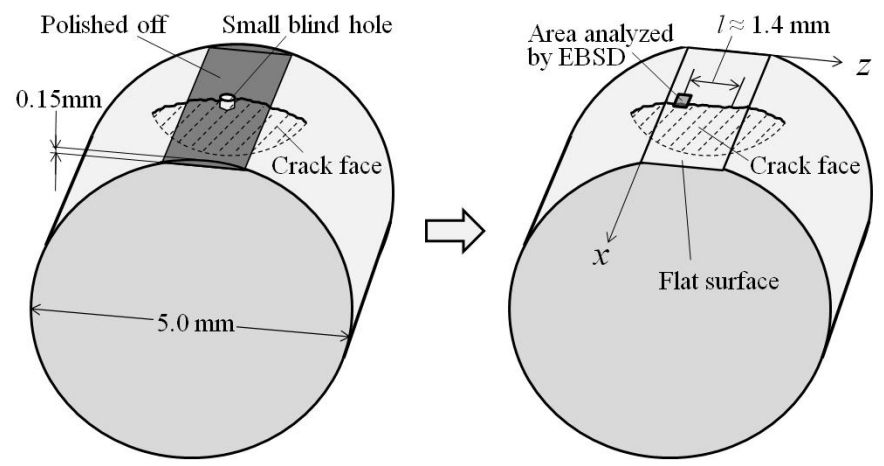

Figure 5: Illustration showing the production of a flat surface in the round-bar specimens and EBSD-analyzed area.

Regarding the microstructures subjected to two-step stressing, L-HB stressing produced moderately coarsened grains ranging from a few to a few tens of micrometers, and few grains remained with the original sub-micron sizes (Fig.6(c)). In H-LB stressing (Fig. 6(d)), on the contrary, the grain coarsening occurred in limited areas of microstructure. Most parts of microstructures

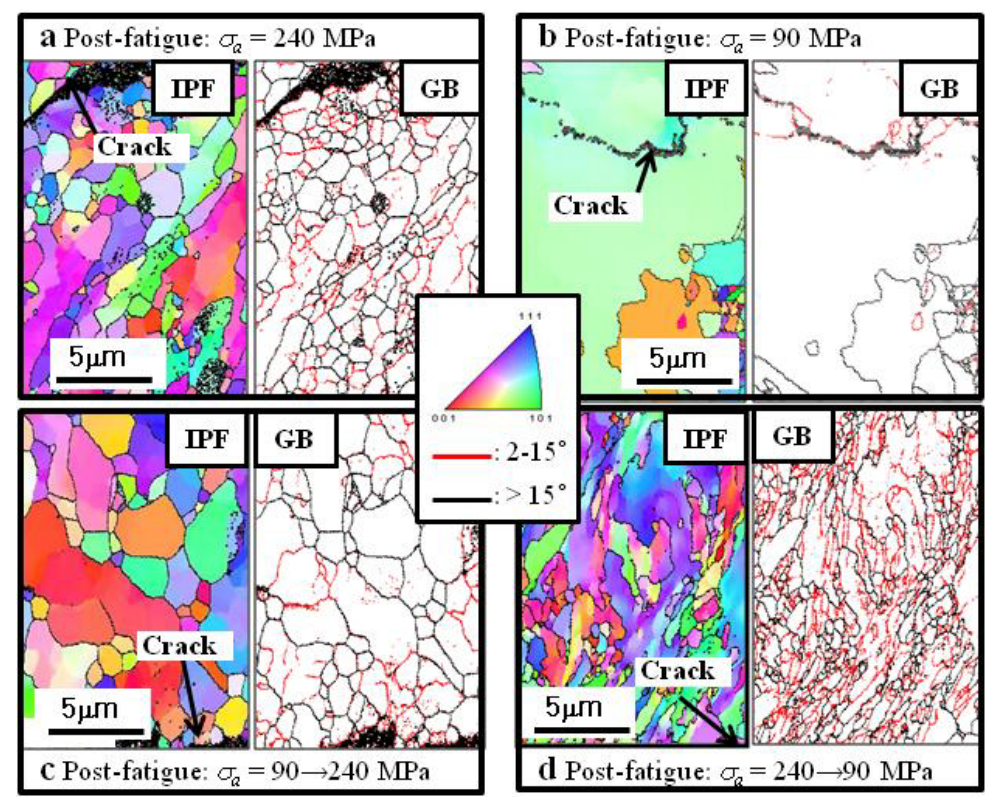

Figure 6: The OIM orientation maps of areas adjacent to crack paths: (a) $\sigma_{a}=240 \mathrm{MPa}$, (b) $\sigma_{a}=90 \mathrm{MPa}$, (c) L-HB stressing, (d) H-LB stressing. 
consisted of grains with the original sub-micron size, and the remainder had coarse grains which contain subgrains isolated with LAGBs.

Fig. 7 shows OM micrographs of the crack growth paths formed under constant fatigue stress tests. The growth direction of a crack depended on the magnitude of applied stress amplitudes. At $\sigma_{a}=240 \mathrm{MPa}$ (Fig. 7(a)), the crack that initiated from the hole grew at $45^{\circ}$ inclination from the loading axis. The inclined crack showed a linear growth path on the macroscale. In LCF tests of UFG metals [11], the $45^{\circ}$ inclined crack growth direction has been commonly observed in the $Z X$-plane. Regarding the crack propagating under $\sigma_{a}=90 \mathrm{MPa}$ (Fig. 7b), however, the macroscale growth direction was perpendicular to the loading axis; nevertheless, the crack grew in a zigzag manner at the microscale. The degree of zigzag growth gradually increased with an increase in crack length.

Fig. 8 shows the crack growth paths under L-HB and H-LB stressing. In LHB stressing (Fig. 8(a)), the crack grew perpendicular to the loading axis under
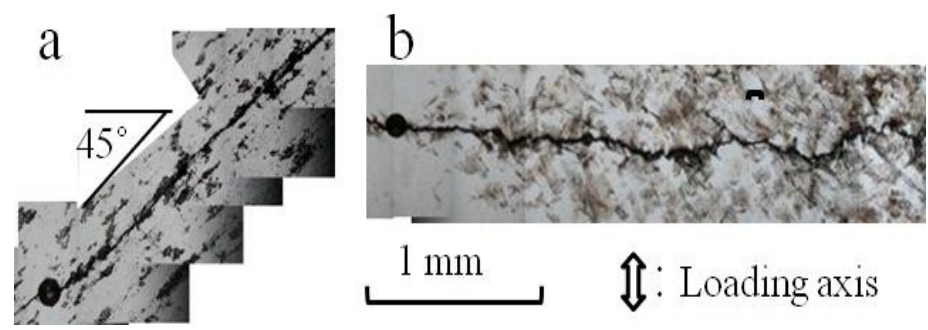

Figure 7: $\quad$ Crack growth paths under constant stress amplitudes; (a) $\sigma_{a}=240$ $\mathrm{MPa}$, (b) $\sigma_{a}=90 \mathrm{MPa}$.

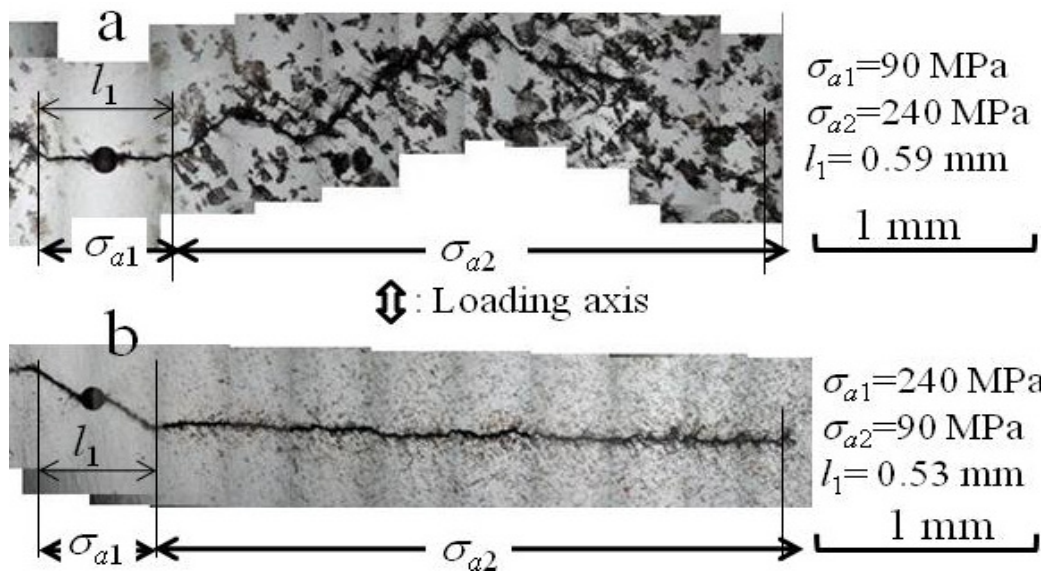

Figure 8: $\quad$ Crack growth paths under two-step stressing; (a) L-HB stressing $\left(\sigma_{a}=90 \rightarrow 240 \mathrm{MPa}\right),(\mathrm{b}) \mathrm{H}-\mathrm{LB}$ stressing $\left(\sigma_{a}=249 \rightarrow 90 \mathrm{MPa}\right)$. 
$\sigma_{a 1}=90 \mathrm{MPa}$ and its length reached approximately $l=0.59 \mathrm{~mm}$ after $2.2 \times 10^{6}$ cycles. Under the second stress $\left(\sigma_{a 2}=240 \mathrm{MPa}\right)$, although the crack paths were nearly perpendicular to the loading axis on the macroscale, the crack path had many branches and meanderings, and showed a drastically large zigzag pattern. In H-LB stressing (Fig. 8b), a 0.53-mm-length crack with a growth path at a $45^{\circ}$ incline to the loading axis was formed under $5.3 \times 10^{4}$ repetitions of $\sigma_{a 1}=$ $240 \mathrm{MPa}$. After a stress amplitude change to $\sigma_{a 2}=90 \mathrm{MPa}$, the crack grew nearly perpendicular to the loading axis, leaving a linear crack path with very small deflections, while the crack path under the constant stress of $\sigma_{a}=90 \mathrm{MPa}$ exhibited a comparatively large zigzag pattern (Fig. 7(b)).

Fig. 9 shows an SEM micrograph of the crack path at the point that the stress changed. In L-HB stressing, after the stress change (Fig. 9(a)), a shear crack formed at the major crack tip and grew along the maximum shear stress plane. Further observations indicated that the shear crack stopped propagating after it reached coarse grains. After stopping of the shear crack propagation, the SBs were initiated. After 6,000 second-stress cycles, the SB growth ceased, and the major crack changed its growth direction with a branching, followed by the crack growth with the consecutive processes of shear-crack/SB formation and crack branching. Consequently, the drastically large zigzag paths were attributed to a repetition of the crack-branching caused by SB formation, blocking growth at coarse grains and the growth along HAGBs of the coarse grains. Fatigue cracks propagate preferentially along HAGBs since they comprise weaker cohesion in their lattice structure [14]. In H-LB stressing (Fig. 9(b)), the surface at the crack edges formed under high stress was comparatively flat, whereas the surface that formed under low stress had traces of localized plastic deformations, suggesting change in the crack growth mechanism under high and low stresses. The crack growth direction just after the stress change under H-LB stressing was estimated by the local maximum tangential normal stress $\left(\sigma_{\theta \max }\right)$ [15], showing the growth direction along the plane of $\sigma_{\theta \max }$ under the subsequent low stress amplitudes.

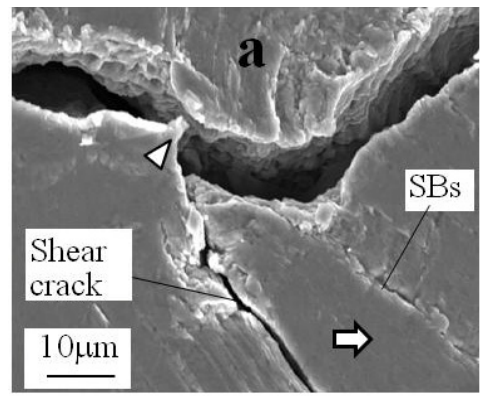

$\triangleleft$ : Crack tip just before the stress change,

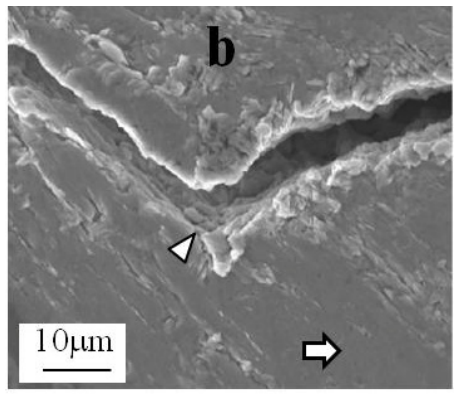

Crack growth direction

Figure 9: The SEM micrographs of the crack paths at the point that the stress changed; (a) L-HB stressing, (b) H-LB stressing. 
In conclusion, the crack at high-stress amplitudes grows along the direction at an incline of $45^{\circ}$ to the loading axis, because of the shear banding induced by the maximum shear stress and the SB decohesion process [16]. At low stress, the crack propagates via the striation formation mechanism, which is associated with crack tip retardation and blunting, because SB formation and decohesion are suppressed under low stresses below a threshold value.

\section{Conclusions}

The main findings of this study can be summarized as follows:

(1) At constant high-stress amplitudes, the crack grew along the direction at an incline of $45^{\circ}$ to the loading axis because of the shear banding induced by the maximum shear stress and the SB decohesion process.

(2) At constant low-stress amplitude, the crack propagated via the striation formation mechanism, which was associated with crack tip retardation and blunting. This was because SB formation and decohesion that occurred at high stresses were suppressed under low stresses below a threshold value. The cracks grew nearly perpendicular to the loading axis in a macro scale with large zigzag manner as a result of the occurrence of dynamically recrystallized grains in excess of $100 \mu \mathrm{m}$.

(3) At L-HB stressing, the macroscopic direction of crack paths before and after the stress change was nearly perpendicular to the loading axis. But, the crack path with small meandering under low-stress amplitude developed with a significantly large zigzag pattern under the subsequent high-stress loading. Coarse grains evolved under the first low-stress repetitions gave rise to the significantly large zigzag paths under the second high-stress repetitions.

(4) At H-LB stressing, a $45^{\circ}$ inclined shear-mode growth path under the first high-stress amplitude turned into a path perpendicular to the loading axis after the stress change, because SB formation and decohesion are suppressed under low stresses.

\section{Acknowledgements}

This study was supported by a Grant-in-Aid (23560093) for Scientific Research (C) from the Ministry of Education, Science and Culture of Japan, as well as the National Research Foundation of Korea (NRF) grant funded by the Korean government (MEST) (No. 2011-0030801), and by a grant from Integrated Technology of Industrial Materials funded by the Ministry of Knowledge Economy, Republic of Korea.

\section{References}

[1] Segal, V.M., Materials processing by simple shear. Mater. Sci. Eng., A197, pp. 157-164, 1995. 
[2] Valiev, R.Z., Structure and mechanical properties of ultrafine-grained metals. Mater. Sci. Eng., A 234-236, pp. 59-66, 1997.

[3] Agnew, S.R., Weertman, J.R., Cyclic softening of ultrafine grain copper. Mater. Sci. Eng., A244, pp. 145-153, 1998.

[4] Vinogradov, A., Hashimoto, S., Multiscale phenomena in fatigue of ultrafine grain materials-an overview. Mater Trans, 42, pp. 74-84, 2000.

[5] Höppel, H.W., Zhou, Z.M., Mughrabi, H., Valiev, R.Z., Microstructural study of the parameters governing coarsening and cyclic softening in fatigued ultrafine-grained copper. Philos Mag -A, 82, pp. 1781-1794, 2002.

[6] Wu, S.D., Wang, Z.G., Jiang, C.B., Li, G.Y., Alexandrov, I.V., Valiev, R.Z., The formation of PSB-like shear bands in cyclically deformed ultrafine grained copper processed by ECAP. Scripta Mater., 48, pp. 16051609, 2003.

[7] Mughrabi, H., Höppel, H.W., Kautz, M., Fatigue and microstructure of ultrafine-grained metals produced by severe plastic deformation. Scripta Mater., 51, pp. 807-812, 2004.

[8] Goto, M., Han, S.Z., Euh, K., Kang, J-H., Kim, S.S., Kawagoishi, N., Formation of a high-cycle fatigue fracture surface and a crack growth mechanism of ultrafine grained copper with different stages of microstructural evolution. Acta Materiala, 58, pp. 6294-6305, 2010.

[9] Vinogradov, A., Nagasaki, S., Patlan, V., Kitagawa, K., Kawazoe, M., Fatigue properties of $5056 \mathrm{Al}-\mathrm{Mg}$ alloy produced by equal-channel angular pressing. Nano Struct. Mater., 11, pp.925-934, 1999.

[10] Kim, H.K., Choi, M-I., Chung, C.S., Shin, D.H., Fatigue properties of ultrafine grained low carbon steel produced by equal channel angular pressing. Mater. Sci. Eng., A340, pp. 243-250, 2003.

[11] Zhang, Z.F., Wu, D.S., Li, Y.J., Liu, S.M., Wang, Z.G., Cyclic deformation and fatigue properties of $\mathrm{Al}-0.7$ wt.\% $\mathrm{Cu}$ alloy produced by equal channel angular pressing. Mater. Sci. Eng., A412, pp. 279-286, 2005.

[12] Valiev, R.Z., Kozlov, E.V., Ivanov, Yu. F., Lian, J., Nazarov, A.A., Baudelet, B., Deformation behaviour of ultra-fine grained copper. Acta Metall. Mater., 42, pp. 2467-2475, 1994.

[13] Goto, M., Han, S.Z., Yakushiji, T., Kim, S.S., Lim, C.Y., Fatigue strength and formation behavior of surface damage in ultrafine grained copper with different non-equilibrium microstructures. Inter. J. Fatigue, 30, pp. 13331344, 2008.

[14] Zhang, Z.F., Wang, Z.G., Comparison of fatigue cracking possibility along large- and low-angle grain boundaries. Mater. Sci. Eng., A284, pp. 285291, 2000.

[15] Erdogan, F., Sih, G.C., On the crack extension in plates under plane loading and transverse shear. Trans. ASME, D85, pp. 519-527, 1963.

[16] Zhang, J.Z., A shear band decohesion model for small fatigue crack growth in an ultra-fine grain aluminium alloy. Eng. Fract. Mech., 65, pp. 665-681, 2000 . 